\title{
Equipartition of the Eigenfunctions of Quantized Ergodic Maps on the Torus
}

\author{
A. Bouzouina ${ }^{\star}$, S. De Bièvre ${ }^{\star}$ \\ Laboratoire de Physique Théorique et Mathématique Université Paris VII, 2 place Jussieu F-75251 \\ Paris Cedex 05, France
}

Received: 8 April 1995

\begin{abstract}
We give a simple proof of the equipartition of the eigenfunctions of a class of quantized ergodic area-preserving maps on the torus. Examples are the irrational translations, the skew translations, the hyperbolic automorphisms and some of their perturbations.
\end{abstract}

\section{Introduction}

Perhaps the simplest trace of the ergodicity of a Hamiltonian dynamical system one expects to find in the corresponding quantum system is the equipartition of its eigenfunctions in the classical limit. Such a phenomenon has been proved to occur in several cases. For the geodesic flow on compact Riemannian manifolds it is proved in [Sc, Z1, CdV]; for Hamiltonian flows on $\mathbb{R}^{2 n}$ in [HMR] and for smooth convex two-dimensional ergodic billiards in [GL].

In this paper we study the quantization and the classical limit of certain area-preserving ergodic maps on the two-torus $T^{(2)}$, viewed as phase space, with canonical coordinates $(q, p) \in[0, a[\times[0, b[$. We will present a rather large class of models for which the desired equipartition result can be proved very easily. We will use the original idea of $[\mathrm{Z} 1, \mathrm{CdV}]$ which can be applied here with considerably less technical complications. Before doing so, we nevertheless first need to decide how to "quantize" an area-preserving map on the torus.

In Sect. 2 we describe the quantum Hilbert spaces associated to the torus. This problem has been addressed and solved by many authors before us with various different approaches [HB, BV, DE, DBDEG], always with the same result. The quantum Hilbert space is an $N$-dimensional complex vector space where $N$ is related to $\hbar$ via the prequantum condition: $2 \pi \hbar N=a b$. It carries an irreducible unitary representation of the discrete Weyl-Heisenberg group $\left\{\left(m \frac{a}{N}, n \frac{b}{N}, \phi\right) \in \mathbb{R}^{3} \mid n, m \in \mathbb{Z}\right\}$. Here we give a rigorous version of the ideas of $[\mathrm{HB}]$ and $[\mathrm{BV}]$, which is

^ CEREMADE, Université Paris-Dauphine, Place du Maréchal de Lattre de Tassigny, 75775 Paris Cedex 16. E-mail: bouzoui@ccr.jussieu.fr.

$\star \star$ UFR de Mathématiques, Université Paris VII. E-mail: debievre@mathp7.jussieu.fr. 
particularly well adapted for our purposes as will be seen below. The approach is based on the following idea. Since the system is one-dimensional, its "wave functions" have to be tempered distributions on the line and since it lives on the torus, they have to be periodic of period $a$ in position and of period $b$ in momentum. It then follows easily that the resulting wave functions form an $N$-dimensional vector subspace $\mathscr{S}^{\prime}(\kappa, N)$ of $\mathscr{S}^{\prime}(\mathbb{R})$ provided $2 \pi \hbar N=a b$ for some $N \in \mathbb{N}^{*}$, and that it is empty otherwise. Here $\kappa \in\left[0, \frac{2 \pi}{a}\left[\times\left[0, \frac{2 \pi}{b}[\right.\right.\right.$ is a parameter related to a choice of phase. We then equip $\mathscr{S}^{\prime}(\kappa, N)$ with a unique Hilbert space structure by requiring that the translations act unitarily. We write $\mathscr{H}_{N}(\kappa)$ for the resulting Hilbert space. The $\mathscr{H}_{N}(\kappa)$ are the quantum Hilbert spaces. The restriction of the representation of the discrete Weyl-Heisenberg group on $\mathscr{S}^{\prime}(\mathbb{R})$ to $\mathscr{H}_{N}(\kappa)$ is unitary and irreducible, the representations for different $\kappa$ being inequivalent. In this sense we obtain a family of inequivalent quantizations. These results are implicit in [HB] and [BV] (for $\kappa=0$ ), and are obtained also in [DE] and [DBDEG], from two different points of view. We feel our approach here has a triple appeal. It uses only one intuitively clear and very simple hypothesis on the quantum states. It uses only the simplest mathematical structures. Finally, and most importantly, it realizes the quantum Hilbert spaces in a way which reduces all semi-classical estimates needed to simple known estimates, as will be seen in Sect. 3 .

In Sect. 3 we show how to quantize observables, i.e. how to associate to each $f \in C^{\infty}\left(T^{(2)}\right)$ an operator on the quantum Hilbert space. We treat both the Weyl and anti-Wick quantizations and show some estimates on their classical limit, needed in the rest of the paper. The Weyl quantization $O p_{\kappa}^{W}(f)$ of $f$ on $\mathscr{H}_{N}(\kappa)$ we present here is identical to the one proposed in [HB] and [DEGI]. The anti-Wick quantization $O p_{\kappa}^{A W}(f)$ is based on a construction of coherent states adapted to the present situation: they are just periodicized Gaussians. These have been used in the literature before by [LV]. We show how some of their expected properties (resolution of the identity, semi-classical behaviour) are readily obtained by remarking that $O p_{\kappa}^{A W}(f)$ is the restriction to $\mathscr{H}_{N}(\kappa)$ of the anti-Wick quantization $O p^{A W}(f)$ of $f$ on $\mathscr{S}^{\prime}(\mathbb{R})$. For completeness, we indicate how they can be used to identify $\mathscr{H}_{N}(\kappa)$ with a reproducing kernel subspace $\mathscr{B}_{N}(\kappa)$ of $L^{2}\left(T^{(2)}, \frac{d q d p}{2 \pi \hbar}\right)$, the so-called Bargmann representation. This is also the Hilbert space one would obtain from geometric quantization, if working with a Kählerian polarization and is as such a space of theta-functions. We have found it convenient to do all estimates in $\mathscr{H}_{N}(\kappa)$ directly. The computations needed to control the behaviour of the thetafunctions when $\hbar$ goes to 0 , although intuitively clear, seem to become rapidly messy.

At this point we can then quantize Hamiltonian flows with Hamiltonian $H \in C^{\infty}\left(T^{(2)}\right)$ by constructing on $\mathscr{H}_{N}(\kappa)$ the unitary one-parameter groups $\exp \left(-i \frac{t}{\hbar} O p_{\kappa}^{W}(H)\right)$. Of course, they are never ergodic. We can also quantize kicked (or pulsed) systems such as the kicked Harper model or the standard map. They have propagators of the form $\exp \left(-\frac{i}{\hbar} O p_{\kappa}^{W}\left(H_{1}\right)\right) \exp \left(-\frac{i}{\hbar} O p_{\kappa}^{W}\left(H_{2}\right)\right)$. We are not aware of any examples of this type that are ergodic. This leaves the question on how to quantize those area-preserving maps known to be ergodic.

The simplest ergodic transformations of the torus are undoubtedly the irrational rotations and the skew translations [CFS]. They were quantized in [DBDEG]. In Sect. 4 we will give a very short proof of the equipartition of their eigenfunctions (Theorem 4.2). Indeed, thanks to the unique ergodicity of these transformations, and using a simple estimate on the anti-Wick quantization, the result is obtained directly from an Egorov theorem, proven in Sect. 4 (Proposition 4.1). This first 
example has the benefit of showing the essence of the arguments needed in the general case.

More generally, suppose $\Phi$ is an ergodic area-preserving map that we know how to quantize. Let us write $M_{\kappa}(\Phi)$ for its quantization: $M_{\kappa}(\Phi)$ is a unitary map on $\mathscr{H}_{N}(\kappa)\left(M_{\kappa}(\Phi) \in \mathfrak{U}\left(\mathscr{H}_{N}(\kappa)\right)\right.$. Suppose moreover one can show an Egorov theorem, i.e. $\forall f \in C^{\infty}\left(T^{(2)}\right), \forall k \in \mathbb{N}, \exists C>0$ so that for all $N$,

$$
\left\|M_{\kappa}(\Phi)^{-k} O p_{\kappa}^{W}(f) M_{\kappa}(\Phi)^{k}-O p_{\kappa}^{W}\left(f \circ \Phi^{k}\right)\right\|_{\mathscr{L}\left(\mathscr{H}_{N}(\kappa)\right)} \leqq \frac{C}{N} .
$$

This means that the classical and quantum evolutions commute " $\bmod O\left(\frac{1}{N}\right)$." It is then a simple matter to adapt the arguments of $[\mathrm{Z} 1, \mathrm{CdV}, \mathrm{HMR}]$ to show an equipartition result as follows (Sect. 5).

Theorem 1.1. Let $\Phi$ be an ergodic area-preserving map on the torus such that $\forall N \exists \kappa \in\left[0, \frac{2 \pi}{a}\left[\times\left[0, \frac{2 \pi}{b}\left[\right.\right.\right.\right.$ and $M_{\kappa}(\Phi) \in \mathfrak{U}\left(\mathscr{H}_{N}(\kappa)\right)$ satisfying (1.1). Write

$$
M_{\kappa}(\Phi) \phi_{j}^{N}=\lambda_{j}^{N} \phi_{j}^{N}
$$

for the eigenvalues and the eigenfunctions of $M_{\kappa}(\Phi)$. Then there exists $E(N) \subset$ $\{1, \ldots, N\}$ satisfying $\lim _{N \rightarrow \infty} \frac{\# E(N)}{N}=1$ such that for all functions $f \in C^{\infty}\left(T^{(2)}\right)$ and for all maps $j: N \in \mathbb{N} \rightarrow j(N) \in E(N)$, we have

and

$$
\lim _{N \rightarrow \infty}\left\langle\phi_{j(N)}^{N}, O p_{\kappa}^{W}(f) \phi_{j(N)}^{N}\right\rangle_{(\kappa, N)}=\int_{T^{(2)}} f(q, p) \frac{d q d p}{a b}
$$

$$
\lim _{N \rightarrow \infty}\left\langle\phi_{j(N)}^{N}, O p_{\kappa}^{A W}(f) \phi_{j(N)}^{N}\right\rangle_{(\kappa, N)}=\int_{T^{(2)}} f(q, p) \frac{d q d p}{a b}
$$

uniformly with respect to the map $j$.

The proof of this theorem is given in Sect. 5. Equation (1.2) and (1.3) can be interpreted as saying that the Wigner and the Husimi distribution of the $\phi_{j(N)}^{N}$ converge to 1 on $T^{(2)}$ (Proposition 3.4, Proposition 3.7). Of course, this is a convergence in the sense of the distributions, not a pointwise convergence.

The diffeomorphisms of the torus known to be ergodic with respect to the Lebesgue measure include, in addition to the translations and the skew translations, the hyperbolic automorphisms and their perturbations. The hyperbolic automorphisms of the torus are the simplest examples of hyperbolic dynamical systems. A particular subclass of them was quantized in [HB]. The general case was solved in [DE] by group theoretic methods and in [DBDEG] through geometric quantization. We treat the general case in Sect. 6 very simply in the spirit of [HB] using the approach of Sect. 2 as follows. Any automorphism $A$ of the torus acts on $\mathbb{R}^{2}$ by a linear symplectic map. The corresponding propagator $M(A)$ on $\mathscr{S}^{\prime}(\mathbb{R})$ is well known from standard quantum mechanics or in terms of group theory (the metaplectic representation $[\mathrm{F}])$. We show that for each $A$ and $N$ there exists at least one $\kappa$ so that $M(A) \mathscr{H}_{N}(\kappa) \subset \mathscr{H}_{N}(\kappa)$ and call $M_{\kappa}(A)$ the restriction of $M(A)$ to $\mathscr{H}_{N}(\kappa)$. As mentioned before, this is just the idea of [HB], except that there the choice $\kappa=0$ was made from the beginning, which restricts the family of "quantizable" $A$, as remarked also in [DE]. We think of $M_{\kappa}(A)$ as the quantization of $A$ in $\mathscr{H}_{N}(\kappa)$.

The Egorov result needed to apply Theorem 1.1 with $\Phi=A$ is immediate. Indeed, when $A$ is an automorphism of the torus, it is a property of the Weyl 
quantization $O p_{\kappa}^{W}(f)$ that

$$
O p_{\kappa}^{W}(f \circ A)=M_{\kappa}(A)^{*} O p_{\kappa}^{W}(f) M_{\kappa}(A)
$$

for a suitable choice of $\kappa$, depending on $A$ and possibly on $N$, as explained above. Equation (1.4) states that the quantum and the classical evolutions commute without error term as in (1.1), which is not surprising since it is a well known property of linear area-preserving maps on $T^{*} \mathbb{R}$. As a result of (1.4), the conclusion of Theorem 1.1 holds with $\Phi$ replaced by $A$. The quantization of the toral automorphisms introduced in this paper is isomorphic to the one obtained in [HB, DE, DBDEG]. By this we mean that there exist unitary operators that identify the quantum Hilbert spaces in those different contexts in such a way as to intertwine the irreducible unitary representation of the discrete Weyl-Heisenberg group, the quantizations of the automorphisms, as well as the Weyl-quantization introduced in each case. Recently, a $C^{*}$-algebraic approach to quantum ergodicity was developed [Z2]. As an example of this approach the Toeplitz quantization of the toral automorphisms is constructed and the analog of Theorem 1.1 proven. As remarked in [Z2], it is not completely obvious that the Toeplitz quantization is isomorphic to the above ones.

Our result on the equipartition of the eigenfunctions of the quantized hyperbolic automorphisms of the torus complements the one obtained in [DEGI]. Indeed, there the result was obtained only for a special class of matrices $A$ considered in [HB] (see Sect. 6 below, Eq. (6.7)) and taking the limit along a particular susbsequence $N_{k}$ of primes depending on $A$. On the other hand, given these restrictions, their result is sharper, since they can take $E(N)=\{1, \ldots, N\}$ (see Theorem 1.1). In this sense, their result proves "unique quantum ergodicity" [Sa] meaning that in the limit $N_{k} \rightarrow \infty$ the Wigner function of the eigenfunctions cannot have support on any other invariant set of the dynamics. Of course, one could protest that this is a slightly exaggerated claim, since the sequence $N_{k}$ is density zero in the integers. It is moreover chosen so as to ensure that the degeneracies of all eigenvalues of the quantized automorphisms remain bounded in the limit. It is tempting to speculate that it is precisely when the degeneracies grow that "unique quantum ergodicity" could be violated. This, at least, is what happens in integrable systems, when constructing eigenfunctions concentrating on closed trajectories, rather than on invariant tori [DB]. It would be interesting to see if such a phenomenon persists here, where all periodic trajectories are highly unstable.

The proof in [DEGI] is of a completely different nature as ours: it is based on an explicit construction of all eigenfunctions of $M_{\kappa}(A)$ and uses heavily arithmetic properties of the map $A$. It therefore cannot be applied to other, not explicitly solvable models. On the other hand, it permits to obtain additional results on the limit $N \rightarrow \infty$ of off-diagonal matrix elements $\left\langle\phi_{j(N)}^{N}, O p_{\kappa}^{A W}(f) \phi_{k(N)}^{N}\right\rangle_{(\kappa, N)}(j(N) \neq k(N))$ which are related to the mixing properties of the classical map. Similar results should be accessible with our approach along the lines of [CR].

All the examples we considered so far are obtained by periodization of affine transformations on the plane. On the plane they are obtained by integrating the flow of quadratic Hamiltonians, and as such they are exactly solvable both classically and quantum mechanically. Although the situation is considerably more complex for the quantum problem on the torus (see Sect. 4 and [DEGI]), it is fair to say that these examples are too simple to capture the essence of the general situation.

The only other class of measure preserving smooth transformations of the torus known to be ergodic that we are aware of all the perturbations of the hyperbolic 
toral automorphisms [M, Si]. If $H \in C^{\infty}\left(T^{(2)}\right)$, we write $\Phi_{t}^{H}$ for the corresponding Hamiltonian flow and introduce, for $A \in S L(2, \mathbb{Z}),|\operatorname{Tr} A|>2$,

$$
\Phi=\Phi_{t}^{H} \circ A .
$$

Since $A$ is Anosov, $\Phi$ will also be Anosov and hence ergodic if $t$ is small enough [M]. We define

$$
M_{\kappa}(\Phi)=e^{-\frac{i t}{\hbar} O p_{\kappa}^{W}(H)} \circ M_{\kappa}(A)
$$

In view of (1.4) and the standard Egorov Theorem for $e^{-\frac{i t}{\hbar} O p_{\kappa}^{W}(H)}$ ([Ro] Theorem IV.10), it is clear (1.1) holds. Hence Theorem 1.1 applies to this example.

\section{The Hilbert Space of States}

Following the standard point of view of the physics literature, we will say that the state of a quantum mechanical particle having $\mathbb{R}$ as configuration space, and hence $\mathbb{R}^{2}$ as phase space, is a tempered distribution $\psi \in \mathscr{S}^{\prime}(\mathbb{R})$. When $\psi$ belongs to $L^{2}(\mathbb{R}, d x)$, the state is said to be normalizable. The phase space translation operators

$$
U(q, p, \phi)=e^{-\frac{i}{\hbar} \phi} e^{\frac{i}{\hbar}(p Q-q P)}
$$

with $(Q \psi)(x)=x \psi(x)$ and $(P \psi)(x)=\frac{\hbar}{i} \frac{d \psi}{d x}(x)$ yield a representation of the Weyl-Heisenberg group on $\mathscr{S}^{\prime}(\mathbb{R})$, restricting to a unitary irreducible representation on $L^{2}(\mathbb{R}, d x)[\mathrm{F}]$ :

$$
U(q, p, \phi) U\left(q^{\prime}, p^{\prime}, \phi^{\prime}\right)=U\left(q+q^{\prime}, p+p^{\prime}, \phi+\phi^{\prime}+\frac{1}{2}\left(q p^{\prime}-p q^{\prime}\right)\right) .
$$

If the system has the two-torus $T^{(2)}=\mathbb{R}^{2} / \Gamma$ as phase space, where $\Gamma=\{(n a, m b) \mid$ $\left.(n, m) \in \mathbb{Z}^{2}\right\}\left(a, b \in \mathbb{R}_{*}^{+}\right.$fixed), what should be its quantum states? It is reasonable to require them to have the same periodicity as the underlying classical system i.e.

$$
\begin{aligned}
& U(a, 0,0) \psi=e^{-i \kappa_{1} a} \psi, \\
& U(0, b, 0) \psi=e^{i \kappa_{2} b} \psi
\end{aligned}
$$

for some $\kappa=\left(\kappa_{1}, \kappa_{2}\right) \in\left[0, \frac{2 \pi}{a}\left[\times\left[0, \frac{2 \pi}{b}[\right.\right.\right.$. Here one allows $\kappa \neq 0$ because two distributions $\psi_{1}$ and $\psi_{2}$ satisfying $\psi_{1}=e^{i \alpha} \psi_{2}$ for some $\alpha \in \mathbb{R}$ describe the same state of the system in the standard formulation of quantum mechanics.

The rest of this section is an immediate extension of the approach used in solid state physics when dealing with electrons in a periodic structure (Bloch waves). In that case, only (2.1) is imposed. Adding (2.2) makes the computations slightly more involved, but still straightforward provided the Poisson sum formula is used:

$$
\sum_{t \in \mathbb{Z}} \delta(x-t)=\sum_{k \in \mathbb{Z}} e^{2 \pi i k x}
$$

Some additional arguments are needed to turn these calculations into proofs. As already pointed out, the results of this section are known under various forms, in particular in signal analysis, the theory of the fast Fourier transform, etc. 
Proposition 2.1. There exist $\psi \in \mathscr{S}^{\prime}(\mathbb{R})(\psi \neq 0)$ satisfying (2.1)-(2.2) iff $\exists N \in \mathbb{N}$ so that

$$
2 \pi \hbar N=a b .
$$

In that case the space of solutions $\mathscr{S}^{\prime}(\kappa, N)$ to $(2.1)-(2.2)$ is $N$-dimensional and each $\psi \in \mathscr{S}^{\prime}(\kappa, N)$ can be written uniquely as

$$
\psi(x)=\sqrt{\frac{a}{N}} \sum_{n \in \mathbb{Z}} c_{n} \delta\left(x-\left(\frac{b \kappa_{2}}{2 \pi}+n\right) \frac{a}{N}\right)
$$

for some $c_{n} \in \mathbb{C}$, satisfying

$$
c_{n+N}=e^{i \kappa_{1} a} c_{n} .
$$

Conversely, each $\psi$ of the form (2.4)-(2.5) belongs to $\mathscr{S}^{\prime}(\kappa, N)$.

Notation. From now on $U(q, p):=U(q, p, 0)$.

Proof. The requirement (2.1)-(2.2) implies $U(a, 0) U(0, b) \psi=U(0, b) U(a, 0) \psi$. Since $U(a, 0) U(0, b)=e^{-\frac{l}{\hbar} a b} U(0, b) U(a, 0)$, there exists $N \in \mathbb{N}$ such that $2 \pi \hbar N=a b$.

To prove the rest of the proposition note that (2.2) reads

$$
e^{i \frac{b}{\hbar} x} \psi=e^{i \kappa_{2} b} \psi \quad \text { in } \mathscr{S}^{\prime}(\mathbb{R}),
$$

from which one concludes that $\psi$ is of the form (2.4) (the factor $\sqrt{\frac{a}{N}}$ is chosen for later convenience). Imposing (2.1) yields (2.5). The last statement is obvious.

From now on we always assume (2.3) holds. Let us introduce $\forall j \in \mathbb{Z}$,

$$
e_{j}^{\kappa}(x)=\sqrt{\frac{a}{N}} \sum_{n} e^{i n a \kappa_{1}} \delta\left(x-\frac{a b \kappa_{2}}{2 \pi N}-j \frac{a}{N}-n a\right) .
$$

The $\left\{e_{j}^{\kappa}\right\}_{j=0}^{N-1}$ form a basis of $\mathscr{S}^{\prime}(\kappa, N)$ which allows the identification of $\psi \in$ $\mathscr{S}^{\prime}(\kappa, N)$ with $\left(c_{j}\right)_{j=0}^{N-1} \in \mathbb{C}^{N}$. This is interpreted as the "position representation." Needless to say, one passes via a Fourier transform to the "momentum representation." This amounts to performing a discrete Fourier transform on the coefficients $c_{n}$. We consider $\mathscr{S}^{\prime}(\kappa, N)$ as the space of quantum states of the system. It will be convenient in further computations to notice that $\mathscr{S}^{\prime}(\kappa, N)$ can be obtained by symmetrisation as follows.

Lemma 2.2. Define

$$
S(\kappa): \psi \in \mathscr{S}(\mathbb{R}) \rightarrow S(\kappa) \psi \in \mathscr{S}^{\prime}(\mathbb{R})
$$

by

$$
S(\kappa) \psi=\sum_{m, n}(-1)^{N m n} e^{i\left(\kappa_{1} a n-\kappa_{2} m b\right)} U(n a, m b) \psi .
$$

Then $S(\kappa)$ maps $\mathscr{S}(\mathbb{R})$ onto $\mathscr{S}^{\prime}(\kappa, N)$.

Remark. One can think of the $S(\kappa)$ as "projections" onto $\mathscr{S}^{\prime}(\kappa, N)$. Note that there is some standard group theory hidden here since the $(\kappa, N)$ index all characters of the discrete Weyl-Heisenberg group over $\Gamma$.

Proof. A simple calculation that we omit. 
We will see below (Sect. 6) what is the role of the freedom in the choice of $\kappa$. For now, let us show how to equip $\mathscr{S}^{\prime}(\kappa, N)$ with a natural Hilbert space structure.

\section{Proposition 2.3.}

$$
U(q, p) \mathscr{S}^{\prime}(\kappa, N) \subset \mathscr{S}^{\prime}(\kappa, N)
$$

iff $\exists\left(n_{1}, n_{2}\right) \in \mathbb{Z}^{2}$ so that $(q, p)=\left(n_{1} \frac{a}{N}, n_{2} \frac{b}{N}\right)$;

(ii) There exists a Hilbert space structure $\langle\cdot, \cdot\rangle_{(\kappa, N)}$ on $\mathscr{S}^{\prime}(\kappa, N)$, unique up to normalization, for which all $U\left(n_{1} \frac{a}{N}, n_{2} \frac{b}{N}\right),\left(n_{1}, n_{2}\right) \in \mathbb{Z}^{2}$ are unitary. It is given by

$$
\left\langle\psi, \psi^{\prime}\right\rangle_{(\kappa, N)}=\sum_{j=0}^{N-1} \bar{c}_{j} c_{j}^{\prime}
$$

in the notations of (2.4)-(2.5). We shall denote the resulting Hilbert space by $\mathscr{H}_{N}(\kappa)=\left(\mathscr{S}^{\prime}(\kappa, N),\langle\cdot, \cdot\rangle_{(\kappa, N)}\right)$; have

(iii) Writing $U_{\kappa}\left(\frac{n_{1}}{N} a, \frac{n_{2}}{N} b\right)$ for the restriction of $U\left(\frac{n_{1}}{N} a, \frac{n_{2}}{N} b\right)$ to $\mathscr{S}^{\prime}(\kappa, N)$, we

$$
L^{2}(\mathbb{R}) \cong \int_{0}^{2 \pi / a} \int_{0}^{2 \pi / b} d m(\kappa) \mathscr{H}_{N}(\kappa)
$$

where $d m(\kappa)=\frac{a b}{(2 \pi)^{2}} d \kappa$, and

$$
U\left(\frac{n_{1}}{N} a, \frac{n_{2}}{N} b\right)=\int_{0}^{2 \pi / a} \int_{0}^{2 \pi / b} d m(\kappa) U_{\kappa}\left(\frac{n_{1}}{N} a, \frac{n_{2}}{N} b\right) .
$$

Remark. Note that (2.8)-(2.9) is nothing but the direct integral decomposition into irreducible subrepresentations of the representation of the discrete WeylHeisenberg group $\left\{\left(n_{1} \frac{a}{N}, n_{2} \frac{b}{N}, \phi\right) \mid n_{1}, n_{2} \in \mathbb{Z}, \phi \in \mathbb{R}\right\}$ on $L^{2}(\mathbb{R})$.

Proof. (i) Using (2.4), for all $\psi$ in $\mathscr{S}^{\prime}(\kappa, N)$,

$$
U(q, p) \psi(x)=\sqrt{\frac{a}{N}} e^{i \frac{2 \pi N}{a b} p q} \sum_{n \in \mathbb{Z}} c_{n} e^{i p\left(\kappa_{2}+\frac{2 \pi}{b} n\right)} \delta\left(x-\frac{a b \kappa_{2}}{2 \pi N}-n \frac{a}{N}-q\right) .
$$

Since $\kappa_{1}$ and $\kappa_{2}$ are defined $\bmod \left(\frac{2 \pi}{a}\right)$ and $\bmod \left(\frac{2 \pi}{b}\right)$ respectively, $\mathscr{S}^{\prime}(\kappa, N)$ is invariant under the action of $U(q, p)$ iff there exists $\kappa_{2}^{\prime}=\kappa_{2} \bmod \frac{2 \pi}{b}$ such that

$$
\frac{a b \kappa_{2}}{2 \pi N}+q=\frac{a b \kappa_{2}^{\prime}}{2 \pi N}
$$

and

$$
c_{n+N} e^{i p \frac{2 \pi}{b}(n+N)}=e^{i \kappa_{1} a} c_{n} e^{i p \frac{2 \pi}{b} n} .
$$

Since $c_{n+N}=e^{i \kappa_{1} a} c_{n}$, it follows that $U(q, p) \mathscr{S}^{\prime}(\kappa, N) \subset \mathscr{S}^{\prime}(\kappa, N)$ iff $(q, p)=\left(n_{1} \frac{a}{N}\right.$, $\left.n_{2} \frac{b}{N}\right)$ with $\left(n_{1}, n_{2}\right) \in \mathbb{Z}^{2}$.

(ii) Since

$$
\begin{gathered}
U\left(m \frac{a}{N}, 0\right) e_{j}^{\kappa}=e_{j+m}^{\kappa}, \\
U\left(0, n \frac{b}{N}\right) e_{j}^{\kappa}=e^{i\left(b \kappa_{2}+2 \pi j\right) \frac{n}{N}} e_{j}^{\kappa},
\end{gathered}
$$


and $e_{j+N}^{\kappa}=e^{-i \kappa_{1} a} e_{j}^{\kappa}$, the requirement for $U\left(n_{1} \frac{a}{N}, n_{2} \frac{b}{N}\right)$ to be unitary for all $\left(n_{1}, n_{2}\right) \in \mathbb{Z}^{2}$ leads to the conditions:

$$
\begin{gathered}
\left\|e_{j}^{\kappa}\right\|_{(\kappa, N)}=\left\|e_{k}^{\kappa}\right\|_{(\kappa, N)} \quad \forall 0 \leqq j, k \leqq N-1, \\
\left\langle e_{j}^{\kappa}, e_{k}^{\kappa}\right\rangle_{(\kappa, N)}=0 \quad \forall j \neq k .
\end{gathered}
$$

Hence, for all $\psi=\sum_{j=0}^{N-1} c_{j} e_{j}^{\kappa}$ and $\psi^{\prime}=\sum_{k=0}^{N-1} c_{k}^{\prime} e_{k}^{\kappa},\langle\cdot, \cdot\rangle_{(\kappa, N)}$ is given by (2.7) up to normalization.

(iii) Identifying $\mathscr{H}_{N}(\kappa)$ with $\mathbb{C}^{N}$ as above, we need to prove $L^{2}(\mathbb{R}) \cong$ $L^{2}\left(\left[0, \frac{2 \pi}{a}\left[\times\left[0, \frac{2 \pi}{b}\left[, d m(\kappa) ; \mathbb{C}^{N}\right)\right.\right.\right.\right.$. For that purpose, we define

$$
F: \psi \in \mathscr{S}(\mathbb{R}) \rightarrow\left(\left\langle e_{j}^{\kappa}, S(\kappa) \psi\right\rangle_{(\kappa, N)}\right)_{j=0}^{N-1} \in L^{2}\left(\left[0, \frac{2 \pi}{a}\left[\times\left[0, \frac{2 \pi}{b}\left[, d m(\kappa) ; \mathbb{C}^{N}\right)\right.\right.\right.\right.
$$

We will first prove it is an isometry. Indeed, using (2.6), a computation shows

$$
\left\langle e_{j}^{\kappa}, S(\kappa) \psi\right\rangle_{(\kappa, N)}=\sqrt{\frac{a}{N}} \sum_{n} e^{i \kappa_{1} n a} \psi\left(\frac{a b \kappa_{2}}{2 \pi N}+j \frac{a}{N}-n a\right) .
$$

So

$$
\begin{aligned}
\int_{0}^{2 \pi / a} & \int_{0}^{2 \pi / b} d m(\kappa) \sum_{j=0}^{N-1} \overline{\left\langle e_{j}^{\kappa}, S(\kappa) \psi\right\rangle_{(\kappa, N)}}\left\langle e_{j}^{\kappa}, S(\kappa) \psi\right\rangle_{(\kappa, N)} \\
= & \frac{a^{2} b}{(2 \pi)^{2} N} \int_{0}^{2 \pi / b} d \kappa_{2} \sum_{j=0}^{N-1} \sum_{n} \sum_{n^{\prime}} \bar{\psi}\left(\frac{a b \kappa_{2}}{2 \pi N}+j \frac{a}{N}-n a\right) \\
& \times \psi\left(\frac{a b \kappa_{2}}{2 \pi N}+j \frac{a}{N}-n^{\prime} a\right) \int_{0}^{2 \pi / a} e^{i \kappa_{1}\left(n^{\prime}-n\right) a} d \kappa_{1} \\
= & \frac{a b}{2 \pi N} \sum_{j=0}^{N-1} \sum_{n} \int_{0}^{2 \pi / b}\left|\psi\left(\frac{a b \kappa_{2}}{2 \pi N}+j \frac{a}{N}-n a\right)\right|^{2} d \kappa_{2}=\int_{\mathbb{R}}|\psi(x)|^{2} d x
\end{aligned}
$$

where we have used the Fubini theorem. Thus we see that $F$ is well defined and has a unique extension to an isometry on $L^{2}(\mathbb{R})$.

To prove that $F$ is onto, we compute $F^{*}$. Note that for all $x \in \mathbb{R}$ there exists unique $n \in \mathbb{Z}, j \in\{0, \ldots, N-1\}, \kappa_{2} \in\left[0, \frac{2 \pi}{b}[\right.$ such that

$$
x=j \frac{a}{N}+\frac{a b \kappa_{2}}{2 \pi N}-n a .
$$

One defines, for $c \in L^{2}\left(\left[0, \frac{2 \pi}{a}\left[\times\left[0, \frac{2 \pi}{b}\left[, d m(\kappa) ; \mathbb{C}^{N}\right)\right.\right.\right.\right.$,

$$
(G c)(x)=\frac{a}{2 \pi} \sqrt{\frac{a}{N}} \int_{0}^{2 \pi / a} d \kappa_{1} e^{-i \kappa_{1} n a} c_{j}\left(\kappa_{1}, \kappa_{2}\right),
$$

where $x, j, n$ and $\kappa_{2}$ are related by (2.12). An easy computation shows $G=F^{*}$, which proves $(2.8)$.

In view of Theorem XIII.83 in [RS], and since the operators $U_{\kappa}\left(\frac{n_{1}}{N} a, \frac{n_{2}}{N} b\right)$ are unitary for all $\kappa \in\left[0, \frac{2 \pi}{a}\left[\times\left[0, \frac{2 \pi}{b}[,(2.9)\right.\right.\right.$ follows if we prove that for all $\psi \in \mathscr{S}(\mathbb{R})$,

$$
\left(U\left(\frac{n_{1}}{N} a, \frac{n_{2}}{N} b\right) \psi\right)_{\kappa}=U_{\kappa}\left(\frac{n_{1}}{N} a, \frac{n_{2}}{N} b\right) S(\kappa) \psi .
$$


This fact is obvious since $U\left(\frac{n_{1}}{N} a, \frac{n_{2}}{N} b\right)$ and $S(\kappa)$ commute. The operators $U_{\kappa}\left(\frac{n_{1}}{N} a, \frac{n_{2}}{N} b\right)$ are the fibers of $U\left(\frac{n_{1}}{N} a, \frac{n_{2}}{N} b\right)$.

The space used in [HB] and [BV] is $\mathscr{H}_{N}(0)$ identified with $\mathbb{C}^{N}$ as above. In [S], $\mathscr{H}_{N}\left(\frac{\pi}{a}, \frac{\pi}{b}\right)$ identified with $\mathbb{C}^{N}$ is used, and in [DE, DEGI] all $\mathscr{H}_{N}(\kappa)$, identified with $\mathbb{C}^{N}$, are introduced. We will see in Sect. 6 why it is necessary to allow for $\kappa \neq 0$. In addition, it will become clear in the next section that by considering all $\kappa$ "at once" as in (2.8), the semi-classical estimates become simple.

\section{The Weyl and Anti-Wick Quantizations}

Classical observables are $C^{\infty}$ functions on $T^{(2)}$. Having constructed the Hilbert space of states, we need to show how to associate to each $f \in C^{\infty}\left(T^{(2)}\right)$ an operator on $\mathscr{H}_{N}(\kappa)$. When the phase space is $\mathbb{R}^{2}$ and the Hilbert space $L^{2}(\mathbb{R}, d x)$, there are several such quantizations available. We show how to adapt two of them - the Weyl and the anti-Wick quantizations - to the two-torus $T^{(2)}$. Note first that any $f \in C^{\infty}\left(T^{(2)}\right)$ can be seen as a periodic $C^{\infty}$ function on $\mathbb{R}^{2}$. The Weyl quantization $O p^{W}(f)$ is the operator on $\mathscr{S}^{\prime}(\mathbb{R})$ given by

$$
O p^{W}(f)=\sum_{n, m \in \mathbb{Z}} f_{n, m} e^{i\left(\frac{2 \pi}{a} n Q-\frac{2 \pi}{b} m P\right)}=\sum_{n, m \in \mathbb{Z}} f_{n, m} U\left(m \frac{a}{N}, n \frac{b}{N}\right),
$$

where

$$
f(q, p)=\sum_{n, m \in \mathbb{Z}} f_{n, m} e^{i\left(\frac{2 \pi}{a} n q-\frac{2 \pi}{b} m p\right)}
$$

(see $[\mathrm{F}]$ or $[\mathrm{Ro}])$. It restricts to a bounded operator on $L^{2}(\mathbb{R})$. Since

$$
U(n a, m b) O p^{W}(f) U(n a, m b)^{*}=O p^{W}(f)
$$

it follows from the definition of $\mathscr{H}_{N}(\kappa)$ that

$$
O p^{W}(f) \mathscr{H}_{N}(\kappa) \subset \mathscr{H}_{N}(\kappa) .
$$

We shall write $O p_{\kappa}^{W}(f)$ for the restriction of $O p^{W}(f)$ to $\mathscr{H}_{N}(\kappa)$. In view of Proposition 2.3 (iii) and (3.1), we immediately have

Lemma 3.1. If $f \in C^{\infty}\left(T^{(2)}\right)$ then, as operators on $L^{2}(\mathbb{R})$,

$$
O p^{W}(f)=\int_{0}^{2 \pi / a} \int_{0}^{2 \pi / b} d m(\kappa) O p_{\kappa}^{W}(f)
$$

To interpret our main result (Theorem 1.1) and to facilitate the link with the literature on torus quantization, we now define "the Wigner function of $\phi_{1}, \phi_{2} \in$ $\mathscr{H}_{N}(\kappa)$." There are several different definitions around in the literature [HB, DEGI, $\mathrm{AB}$ ]. Our discussion will elucidate the origin of this apparent freedom of choice.

There are at least two equally elegant and equivalent ways to define the Wigner function of two square integrable functions $\phi_{1}$ and $\phi_{2}$ on the line. We will see that, adapted to the torus, they yield different notions. The first approach is interpretationally the most natural [HOSW]. Given $\phi_{1}, \phi_{2} \in L^{2}(\mathbb{R})$, one defines the tempered 
distribution $W\left(\phi_{1}, \phi_{2}\right)$ on $\mathbb{R}^{2}$ as follows:

$$
(2 \pi \hbar)^{-1} W\left(\phi_{1}, \phi_{2}\right): f \in \mathscr{S}\left(\mathbb{R}^{2}\right) \rightarrow\left\langle\phi_{1}, O p^{W}(f) \phi_{2}\right\rangle_{L^{2}(\mathbb{R})} \in \mathbb{C}
$$

This is often written with the usual abuse of notation as

$$
\left\langle\phi_{1}, O p^{W}(f) \phi_{2}\right\rangle_{L^{2}(\mathbb{R})}=\iint \frac{d q d p}{2 \pi \hbar} W\left(\phi_{1}, \phi_{2}\right) f .
$$

Equation (3.4) is at the origin of the interpretation of $W(\phi, \phi)$ as a "quasiprobability density": the quantum expectation value $\left\langle\phi, O p^{W}(f) \phi\right\rangle_{L^{2}(\mathbb{R})}$ is written as the "average" of $f$ for the "quasi-probability density" $W(\phi, \phi)$ [HOSW]. For the second approach to the definition, recall first that the Weyl quantization is a bijection

$$
f \in \mathscr{S}^{\prime}\left(\mathbb{R}^{2}\right) \rightarrow O p^{W}(f) \in \mathscr{L}\left(\mathscr{S}(\mathbb{R}), \mathscr{S}^{\prime}(\mathbb{R})\right)
$$

([F, Theorem 1.30 and Definition (2.1)]). One calls $f$ the Weyl symbol of $O p^{W}(f)$. Defining for $\phi_{1}, \phi_{2} \in L^{2}(\mathbb{R})$ the rank one operator $P_{\phi_{1}, \phi_{2}}$ by

$$
P_{\phi_{1}, \phi_{2}} \psi=\left\langle\phi_{1}, \psi\right\rangle_{L^{2}(\mathbb{R})} \phi_{2} \quad \forall \psi \in L^{2}(\mathbb{R})
$$

the Wigner function $W\left(\phi_{1}, \phi_{2}\right)$ of $\phi_{1}, \phi_{2}$ can also be defined as the Weyl symbol of $P_{\phi_{1}, \phi_{2}}$. It turns out that these two definitions are equivalent. This is a direct consequence of the following property of the Weyl quantization referred to as unitarity [Ro], traciality [FGBV], or the Moyal identity [F], depending on the authors: Weyl quantization is a unitary map from $L^{2}\left(\mathbb{R}^{2}, \frac{d q d p}{2 \pi \hbar}\right)$ onto the Hilbert-Schmidt operators on $L^{2}(\mathbb{R})$, in particular,

$$
\operatorname{Tr} O p^{W}(g) O p^{W}(f)=\int \frac{d q d p}{2 \pi \hbar} f g, \quad \forall f, g \in L^{2}\left(\mathbb{R}^{2}\right) .
$$

Hence, inserting $O p^{W}\left(W\left(\phi_{1}, \phi_{2}\right)\right)=P_{\phi_{1}, \phi_{2}}=O p^{W}(g)$ into (3.5), the compatibility of both definitions follows from (3.4). Remark that, more generally, (3.5) implies that the Weyl symbol of any Hilbert-Schmidt operator $G$ on $L^{2}(\mathbb{R})$ is the tempered distribution $f \in \mathscr{S}\left(\mathbb{R}^{2}\right) \rightarrow 2 \pi \hbar \operatorname{Tr} G O p^{W}(f) \in \mathbb{C}$. How can this be adapted to the torus? We start with the following definition.

Definition 3.2. Let $G \in \mathscr{L}\left(\mathscr{H}_{N}(\kappa)\right)$. The Weyl symbol of $G$ is the distribution $\sigma_{\kappa}^{W}(G)$, defined by:

$$
\sigma_{\kappa}^{W}(G): f \in C^{\infty}\left(T^{(2)}\right) \rightarrow \frac{a b}{N} \operatorname{Tr} G O p_{\kappa}^{W}(f) .
$$

This seems fair enough in view of the above. But be careful: a simple calculation shows that $\forall f \in C^{\infty}\left(T^{(2)}\right), \sigma_{\kappa}^{W}\left(O p_{\kappa}^{W}(f)\right) \neq f$ (see (3.8) below). This might seem annoying but is unavoidable: the linear map $f \in C^{\infty}\left(T^{(2)}\right) \rightarrow O p_{\kappa}^{W}(f) \in$ $\mathscr{L}\left(\mathscr{H}_{N}(\kappa)\right)$ is not injective since it maps an infinite dimensional space to a finite dimensional one. As a result, one could at best call $f$ "a" Weyl symbol of $O p_{\kappa}^{W}(f)$, never "the" Weyl symbol. In the spirit of (3.3)-(3.4) and Definition 3.2, we now have: 
Definition 3.3. The Wigner function of $\phi_{1}, \phi_{2} \in \mathscr{H}_{N}(k)$ is the distribution $W_{\kappa}\left(\phi_{1}, \phi_{2}\right)$ :

$$
W_{\kappa}\left(\phi_{1}, \phi_{2}\right): f \in C^{\infty}\left(T^{(2)}\right) \rightarrow \frac{a b}{N}\left\langle\phi_{1}, O p_{\kappa}^{W}(f) \phi_{2}\right\rangle_{(\kappa, N)}
$$

or $\forall f \in C^{\infty}\left(T^{(2)}\right)$,

$$
\left\langle\phi_{1}, O p_{\kappa}^{W}(f) \phi_{2}\right\rangle_{(\kappa, N)}=\int_{T^{(2)}} \frac{d q d p}{2 \pi \hbar} W_{\kappa}\left(\phi_{1}, \phi_{2}\right) f .
$$

This definition coincides with the one given in [HB] for $\kappa=0$, as a computation shows. It is actually easy to see that $W_{\kappa}\left(\phi_{1}, \phi_{2}\right)$ and, more generally, the Weyl symbol of any $G \in \mathscr{L}\left(\mathscr{H}_{N}(\kappa)\right)$ is of the form

$$
\sigma_{\kappa}^{W}(G)=\sum_{j, k=1}^{2 N} c_{j, k}(G) \delta\left(q-q_{j}(\kappa)\right) \delta\left(p-p_{k}(\kappa)\right)
$$

for some $c_{j, k}(G) \in \mathbb{C}$ and where $q_{j}(\kappa)=\left(\frac{j}{2}-\frac{a \kappa_{1}}{2 \pi}\right) \frac{a}{N}, \quad p_{k}(\kappa)=\left(\frac{k}{2}-\frac{b \kappa_{2}}{2 \pi}\right) \frac{b}{N}$. Note that the space of distributions of the form (3.8) is $(2 N)^{2}$-dimensional, whereas $\mathscr{L}\left(\mathscr{H}_{N}(\kappa)\right)$ is only $N^{2}$-dimensional. This explains why not all $c_{j, k}(G)$ are independent: $c_{j, k}(G), c_{j+N, k}(G), c_{j, k+N}(G)$ and $c_{j+N, k+N}(G)(j, k=0, \ldots, N-1)$ are all equal up to a sign, as remarked already in [HB] for $\kappa=0$. This makes the Weyl symbol of $G$ a somewhat unpleasant object to work with. In spite of this and thanks to (3.7), it is easy to rephrase Theorem 1.1 in terms of the Wigner function of the $\phi_{j(N)}^{N}$.

Proposition 3.4. Under the hypotheses of Theorem 1.1,

$$
N W_{\kappa}\left(\phi_{j(N)}^{N}, \phi_{j(N)}^{N}\right) \stackrel{N \rightarrow \infty}{\longrightarrow} 1
$$

in the sense of distributions on $T^{(2)}$.

In [DEGI] a different definition of the Wigner function is used. It is inspired by the second definition for the Wigner function on the line. In our formalism, it can be understood as follows. We already remarked that the map $f \rightarrow O p_{\kappa}^{W}(f)$ is not injective. Let us show it is surjective. For that purpose, consider the $N^{2}$-dimensional Hilbert subspace $\mathscr{I}_{N}$ of $L^{2}\left(T^{(2)}, \frac{d q d p}{a b}\right)$ made up of all trigonometic polynomials $f(q, p)=\sum_{r, s=0}^{N-1} f_{r, s} e^{i\left(\frac{2 \pi}{a} s q-\frac{2 \pi}{b} r p\right)}$.

Lemma 3.5. $O p_{\kappa}^{W}$ is a unitary map from $\mathscr{I}_{N}$ onto $\left.\mathscr{L}\left(\mathscr{H}_{N}(\kappa)\right),\langle\cdot, \cdot\rangle_{H S}\right)$, where $\forall F, G \in \mathscr{L}\left(\mathscr{H}_{N}(\kappa)\right),\langle F, G\rangle_{H S}=\operatorname{Tr} F^{*} G$. In particular

$$
\forall f, g \in \mathscr{I}_{N}, \quad \operatorname{Tr}\left(O p_{\kappa}^{W}(f)\right)^{*} O p_{\kappa}^{W}(g)=\int_{T^{(2)}} \frac{d q d p}{2 \pi \hbar} \bar{f} g .
$$

Proof. It suffices to establish that the $U\left(r \frac{a}{N}, s \frac{b}{N}\right)(r, s=0, \ldots, N-1)$ form a $\langle\cdot, \cdot\rangle_{H S}$-orthogonal basis of $\mathscr{L}\left(\mathscr{H}_{N}(\kappa)\right)$. This follows from

$$
\operatorname{Tr} U\left(r \frac{a}{N}, s \frac{b}{N}\right)^{*} U\left(r^{\prime} \frac{a}{N}, s^{\prime} \frac{b}{N}\right)=N \delta_{r, r^{\prime}} \delta_{s, s^{\prime}} .
$$

This result suggests the following definition: 
Definition 3.6. Let $F \in \mathscr{L}\left(\mathscr{H}_{N}(\kappa)\right)$. The polynomial Weyl symbol of $F$ is the unique element $f \in \mathscr{I}_{N}$ so that $O p_{\kappa}^{W}(f)=F$. Similarly, the polynomial Wigner function $W_{P}^{\kappa}\left(\phi_{1}, \phi_{2}\right)$ of $\phi_{1}, \phi_{2} \in \mathscr{H}_{N}(\kappa)$ is the unique element of $\mathscr{I}_{N}$ so that $O p_{\kappa}^{W}\left(W_{P}^{\kappa}\left(\phi_{1}, \phi_{2}\right)\right)=P_{\phi_{1}, \phi_{2}}$. One easily sees that

$$
f(q, p)=\frac{1}{N} \sum_{r, s=0}^{N-1} e^{i\left(\frac{2 \pi}{a} s q-\frac{2 \pi}{b} r p\right)} \operatorname{Tr} U\left(r \frac{a}{N}, s \frac{b}{N}\right)^{*} F
$$

and

$$
W_{P}^{\kappa}\left(\phi_{1}, \phi_{2}\right)(q, p)=\frac{1}{N} \sum_{r, s=0}^{N-1}\left\langle\phi_{1}, U\left(-r \frac{a}{N},-s \frac{b}{N}\right) \phi_{2}\right\rangle_{(\kappa, N)} e^{i\left(\frac{2 \pi}{a} s q-\frac{2 \pi}{b} r p\right)} .
$$

Several remarks are in order. First of all, $W_{P}^{\kappa}\left(\phi_{1}, \phi_{2}\right)$ differs from $W_{\kappa}\left(\phi_{1}, \phi_{2}\right)$ for all choices of $\phi_{1}, \phi_{2}$. This is clear from (3.8). Note also that (3.9) means that $W_{P}^{\kappa}\left(\phi_{1}, \phi_{2}\right)$ satisfies

$$
\left\langle\phi_{1}, O p_{\kappa}^{W}(f) \phi_{2}\right\rangle_{(\kappa, N)}=\int_{T^{(2)}} \frac{d q d p}{2 \pi \hbar} W_{P}^{\kappa}\left(\phi_{1}, \phi_{2}\right)(q, p) f(q, p)
$$

for $f \in \mathscr{I}_{N}$ but not for all $f \in C^{\infty}\left(T^{(2)}\right)$. One nevertheless has the following result:

Proposition 3.7. Under the hypotheses of Theorem 1.1,

$$
N W_{P}^{\kappa}\left(\phi_{j(N)}^{N}, \phi_{j(N)}^{N}\right) \stackrel{N \rightarrow \infty}{\longrightarrow} 1
$$

in the sense of distributions on $T^{(2)}$.

Proof. For $f \in C^{\infty}\left(T^{(2)}\right)$, write $f=f_{N}+f_{R}$, where $f_{N}$ is the projection of $f$ onto $\mathscr{I}_{N}$. Then, since $W_{P}^{\kappa} \in \mathscr{I}_{N}$,

$$
\begin{aligned}
N \int_{T^{(2)}} & \frac{d q d p}{a b} f(q, p) W_{P}^{\kappa}\left(\phi_{j(N)}^{N}, \phi_{j(N)}^{N}\right)-\int_{T^{(2)}} \frac{d q d p}{a b} f(q, p) \\
& =N \int_{T^{(2)}} \frac{d q d p}{a b} f_{N}(q, p) W_{P}^{\kappa}\left(\phi_{j(N)}^{N}, \phi_{j(N)}^{N}\right)-\int_{T^{(2)}} \frac{d q d p}{a b} f(q, p) \\
& =\left\langle\phi_{j(N)}^{N}, O p_{\kappa}^{W}\left(f_{N}\right) \phi_{j(N)}^{N}\right\rangle-\int_{T^{(2)}} \frac{d q d p}{a b} f(q, p) \\
& =\left\langle\phi_{j(N)}^{N}, O p_{\kappa}^{W}(f) \phi_{j(N)}^{N}\right\rangle-\int_{T^{(2)}} \frac{d q d p}{a b} f(q, p)-\left\langle\phi_{j(N)}^{N}, O p_{\kappa}^{W}\left(f_{R}\right) \phi_{j(N)}^{N}\right\rangle .
\end{aligned}
$$

A simple estimate shows that the last term converges to zero so that Theorem 1.1 implies the result.

The polynomial Wigner function was introduced in [DEGI] (and is simply called the Wigner function there). Polynomial Weyl symbols should a priori be easier to deal with than general Weyl symbols. To see this, remark that any $f \in \mathscr{I}_{N}$ is completely determined by its values on the lattice $\left(q_{r}, p_{s}\right)=\left(r \frac{a}{N}, s \frac{b}{N}\right),(r, s=$ $0, \ldots, N-1)$. Moreover, given $d_{r, s} \in M_{N}(\mathbb{C})$, there is a unique $f \in \mathscr{I}_{N}$ so that $f\left(r \frac{a}{N}, s \frac{b}{N}\right)=d_{r, s}$, and hence in view of Lemma 3.5, a unique $F \in \mathscr{L}\left(\mathscr{H}_{N}(\kappa)\right)$ so 
that $F=O p_{\kappa}^{W}(f)$. There are now no constraints between the matrix elements $d_{r, s}$ as in the case of the Weyl symbol. As we point out in Sect. $6, W_{P}^{\kappa}$ has some drawbacks as well, in particular for the study of the quantization of toral automorphisms. There are still other definitions of "Wigner function" around in the literature $[\mathrm{AB}]$. They are obtained by taking the explicit expression for $W\left(\phi_{1}, \phi_{2}\right)(q, p)$ for $\phi_{1}, \phi_{2} \in$ $\mathscr{S}(\mathbb{R})$ and by trying to adapt it to the torus. The result has no obvious link with Weyl quantization and we shall not consider it further.

To describe the anti-Wick quantization we need to fix some notations. For $z \in \mathbb{C}$, and $\operatorname{Im} z>0$, define the gaussian

$$
\eta_{0,0, z}(x)=\left(\frac{\operatorname{Im} z}{\pi \hbar}\right)^{\frac{1}{4}} e^{\frac{i}{2 \hbar} z x^{2}}
$$

We can then construct the coherent states [Pe]

$$
\eta_{q, p, z}(x)=\left(U(q, p) \eta_{0,0, z}\right)(x)
$$

in the standard manner. It will be useful on occasion to use the bra-ket notation of Dirac:

$$
\eta_{q, p, z}(x)=\langle x \mid q, p, z\rangle \text {. }
$$

Using the symmetrisation operator $S(\kappa)$ introduced in Sect. 2, we define

$$
|q, p, z, \kappa\rangle=S(\kappa)|q, p, z\rangle \in \mathscr{H}_{N}(\kappa) .
$$

We are now ready to define, for any $f \in L^{\infty}\left(T^{(2)}\right)$ its anti-Wick quantization by

$$
O p_{\kappa}^{A W}(f)=\int_{0}^{a} \int_{0}^{b} \frac{d p d q}{2 \pi \hbar} f(q, p)|q, p, z, \kappa\rangle\langle q, p, z, \kappa| .
$$

This is of course the obvious equivalent of the usual anti-Wick quantization on $L^{2}(\mathbb{R})$ :

$$
O p^{A W}(f)=\int_{\mathbb{R}^{2}} \frac{d p d q}{2 \pi \hbar} f(q, p)|q, p, z\rangle\langle q, p, z| .
$$

This, we recall, has the property (Resolution of the identity)

$$
O p^{A W}(1)=\operatorname{Id}_{L^{2}(\mathbb{R})}=\int_{\mathbb{R}^{2}} \frac{d p d q}{2 \pi \hbar}|q, p, z\rangle\langle q, p, z| .
$$

\section{Lemma 3.8.}

(i) $\left(O p_{\kappa}^{A W}(f)\right)^{*}=O p_{\kappa}^{A W}(\bar{f})$.

(ii) If $f \in C^{\infty}\left(T^{(2)}\right)$ then

$$
O p^{A W}(f)=\int_{0}^{2 \pi / a} \int_{0}^{2 \pi / b} d m(\kappa) O p_{\kappa}^{A W}(f) .
$$

(iii)

$$
O p_{\kappa}^{A W}(1)=\operatorname{Id}_{\mathscr{H}_{N}(\kappa)} \quad \text { (Resolution of the identity). }
$$

(iv) For all $f \in C^{\infty}\left(T^{(2)}\right)$ and $\kappa$,

$$
\left\|O p_{\kappa}^{A W}(f)\right\|_{\mathscr{L}\left(\mathscr{H}_{N}(\kappa)\right)} \leqq\|f\|_{\infty}
$$


Proof. (i) follows by a simple calculation.

For (ii), we first show that there exists $C>0$ so that

$$
\sup _{\kappa}\left\|O p_{\kappa}^{A W}(f)\right\|_{\mathscr{L}\left(\mathscr{H}_{N}(\kappa)\right)} \leqq C .
$$

Theorem XIII.83 in [RS] then implies that the right-hand side of (3.12) is well defined. To show (3.15) note simply that for all $\phi$ and $\psi$ in $\mathscr{H}_{N}(\kappa)$,

$$
\left\langle\phi, O p_{\kappa}^{A W}(f) \psi\right\rangle_{(\kappa, N)} \leqq\|\phi\|_{(\kappa, N)}\|\psi\|_{(\kappa, N)}\|f\|_{\infty} \int_{T^{(2)}}\langle q, p, z, \kappa \mid q, p, z, \kappa\rangle \frac{d q d p}{2 \pi \hbar} .
$$

Since, as is easily checked, $\langle q, p, z, \kappa \mid q, p, z, \kappa\rangle$ is continuous in $\kappa,(3.15)$ follows. To prove (3.12) it suffices to compute $O p^{A W}(f) \psi$ for $\psi \in \mathscr{S}(\mathbb{R})$. Using the periodicity of $f$ and Proposition 2.3 (iii), we have, writing $\psi_{\kappa}=S(\kappa) \psi$,

$$
\begin{aligned}
O p^{A W}(f) \psi= & \sum_{m, n} \int_{0}^{a} \int_{0}^{b} \frac{d q d p}{2 \pi \hbar} f(q, p)|q+n a, p+m b, z\rangle \\
& \times \int_{0}^{\frac{2 \pi}{a}} \int_{0}^{\frac{2 \pi}{b}} d m(\kappa)\left\langle q+n a, p+m b, z, \kappa \mid \psi_{\kappa}\right\rangle_{(\kappa, N)} .
\end{aligned}
$$

Since, as a simple calculation shows, $U(n a, m b) S(\kappa)=S(\kappa) U(n a, m b)$ we have, using (3.10),

$$
\begin{aligned}
|q+n a, p+m b, z, \kappa\rangle & =S(\kappa) U(n a, m b) e^{\frac{l}{2 \hbar}(n a p-m b q)}|q, p, z\rangle \\
& =(-1)^{N m n} e^{-i\left(\kappa_{1} n a-\kappa_{2} m b\right)} e^{\frac{l}{2 \hbar}(n a p-m b q)}|q, p, z, \kappa\rangle .
\end{aligned}
$$

Then, one obtains using (2.6),

$$
\begin{aligned}
O p^{A W}(f) \psi= & \int_{0}^{2 \pi / a} \int_{0}^{2 \pi / b} d m(\kappa) \int_{0}^{a} \int_{0}^{b} \frac{d q d p}{2 \pi \hbar} f(q, p) \sum_{m, n}(-1)^{N m n} e^{+i\left(\kappa_{1} n a-\kappa_{2} m b\right)} \\
& \times U(n a, m b)|q, p, z\rangle\left\langle q, p, z, \kappa \mid \psi_{\kappa}\right\rangle_{(\kappa, N)} \\
= & \int_{0}^{2 \pi / a} \int_{0}^{2 \pi / b} d m(\kappa) O p_{\kappa}^{A W}(f) \psi_{\kappa} .
\end{aligned}
$$

This proves (3.12). Note that (iii) is a special case of (ii) for $f=1$ and that (iv) follows from (iii).

Remark. Using the resolution of the identity, one can define an isometric map

$$
W(\kappa, z): \psi \in \mathscr{H}_{N}(\kappa) \longrightarrow W(\kappa, z) \psi \in L\left(T^{(2)}, \frac{d q d p}{2 \pi \hbar}\right)
$$

by

$$
(W(\kappa, z) \psi)(q, p)=\langle q, p, z, \kappa \mid \psi\rangle .
$$

The image of $\mathscr{H}_{N}(\kappa)$ under $W(\kappa, z)$ is a reproducing kernel subspace of $L\left(T^{(2)}, \frac{d q d p}{2 \pi \hbar}\right)$. In the language of geometric quantization, it is the space of square integrable polarized sections of a prequantum line bundle over $T^{(2)}$ determined by 
$\kappa$ and $N$ [DBDEG]. Here the polarization is Kählerian and determined by the complex vector field $X_{z}=\partial_{q}+z \partial_{p}$ on $T^{(2)}$. The function $|\langle\cdot, \cdot, z, \kappa \mid \psi\rangle|^{2}$ on $T^{(2)}$ is referred to as the "Husimi distribution of $\psi$ " in the physics literature.

For the proof of the equipartition of the eigenfunctions we need to compare the Weyl and the anti-Wick quantizations as $N \rightarrow \infty$. The simple estimate needed is given by the following lemma.

Lemma 3.9. For all $f \in C^{\infty}\left(T^{(2)}\right)$,

$$
\left\|O p_{\kappa}^{W}(f)-O p_{\kappa}^{A W}(f)\right\|_{\mathscr{L}\left(\mathscr{H}_{N}(\kappa)\right)}=O\left(N^{-1}\right) \quad(N \rightarrow \infty) .
$$

Proof. Thanks to (3.2),(3.12) and Theorem XIII.83 in [RS], we have

$$
\sup _{\kappa}\left\|O p_{\kappa}^{W}(f)-O p_{\kappa}^{A W}(f)\right\|_{\mathscr{L}\left(\mathscr{H}_{N}(\kappa)\right)}=\left\|O p^{W}(f)-O p^{A W}(f)\right\|_{\left.\mathscr{L}_{(L}^{2}(\mathbb{R})\right)} .
$$

It is a standard result [GL, HMR] that

$$
\left\|O p^{W}(f)-O p^{A W}(f)\right\|_{\mathscr{L}\left(L^{2}(\mathbb{R})\right)}=O\left(N^{-1}\right) \quad(N \rightarrow \infty) .
$$

Thus (3.16) is proved.

We end this section with a semi-classical estimate on the Weyl quantization, crucial for the proof of the equipartition result in Sect. 5.

Proposition 3.10. For all $f \in C^{\infty}\left(T^{(2)}\right)$,

$$
\frac{1}{N} \operatorname{Tr} O p_{\kappa}^{W}(f) \stackrel{N \rightarrow \infty}{\longrightarrow} \int_{0}^{a} \int_{0}^{b} \frac{d q d p}{a b} f(q, p) .
$$

Proof. Using the basis defined above,

$$
\frac{1}{N} \operatorname{Tr} O p_{\kappa}^{W}(f)=\frac{1}{N} \sum_{j=0}^{N-1} \sum_{n, m} f_{n, m}\left\langle e_{j}^{\kappa}, U\left(m \frac{a}{N}, n \frac{b}{N}\right) e_{j}^{\kappa}\right\rangle .
$$

By (2.10) and (2.11), we have

$$
\frac{1}{N} \operatorname{Tr} O p_{\kappa}^{W}(f)=\frac{1}{N} \sum_{j=0}^{N-1} \sum_{l, n} \sum_{r=0}^{N-1} f_{n, l N+r} e^{i \frac{\pi}{N}(l N+r) n} e^{-l l \kappa_{1} a} e^{i \frac{2 \pi}{N} n\left(\frac{b \kappa_{2}}{2 \pi}+j+r\right)}\left\langle e_{j}^{\kappa}, e_{j+r}^{\kappa}\right\rangle_{(\kappa, N)} .
$$

Then, since $\left\{e_{j}^{\kappa}\right\}_{j=0}^{N-1}$ is an orthonormal basis,

$$
\begin{aligned}
\frac{1}{N} \operatorname{Tr} O p_{\kappa}^{W}(f) & =\sum_{l, n} f_{n, l N}(-1)^{l n} e^{-i l \kappa_{1} a} e^{i \frac{2 \pi}{N} n \frac{b \kappa_{2}}{2 \pi}} \frac{1}{N} \sum_{j=0}^{N-1} e^{\imath \frac{2 \pi}{N} n j} \\
& =f_{0,0}+\sum_{k, l \in \mathbb{Z}^{*}} f_{k N, l N} e^{i\left(k b \kappa_{2}-l \kappa_{1} a\right)} .
\end{aligned}
$$

The result follows using the regularity of $f$. 


\section{Translations and Skew Translations}

In this section, we study two particular maps which, as stated in the introduction, are undoubtedly the simplest ergodic transformations of the torus: the irrational translations and the skew translations. We will set $a=b=1$ for convenience. The translations are denoted by $\tau_{\alpha}$, where $\alpha=\left(\alpha_{1}, \alpha_{2}\right) \in T^{(2)}$. If $\alpha$ is such that $\alpha_{1} / \alpha_{2} \notin \mathbb{Q}, \tau_{\alpha}$ is said to be irrational. They act on $T^{(2)}$ as $\tau_{\alpha}:(q, p) \in T^{(2)} \rightarrow\left(q+\alpha_{1}, p+\alpha_{2}\right) \in T^{(2)}$. The skew translations are denoted by $\Phi_{\beta}^{k}$, where $\beta \notin \mathbb{Q}, k \in \mathbb{Z} ; \Phi_{\beta}^{k}$ is defined as $\Phi_{\beta}^{k}=\tau_{(0, \beta)} \circ K$ with $K \in S L(2, \mathbb{Z})$ of the form $K=\left(\begin{array}{ll}1 & k \\ 0 & 1\end{array}\right)$. Both $\tau_{\alpha}$ and $\Phi_{\beta}^{k}$ are uniquely ergodic area-preserving maps [CFS], meaning that there exists a unique invariant probability measure for them (the Lebesgue measure). A well known and useful fact concerning uniquely ergodic maps $\Phi$ on a compact metric space $X$ is that for each continuous function $f$ on $X$

$$
\frac{1}{T} \sum_{k=1}^{T} f \circ \Phi^{k} \stackrel{T \rightarrow \infty}{\longrightarrow} m(f) \quad \text { in } L^{\infty}(X)
$$

where $m$ is this unique invariant probability measure [M,CFS]. We will see below how this permits a particularly simple proof of the equipartition property. We first need to show how to associate to each $\tau_{\alpha}$ and $\Phi_{\beta}^{k}$ a unitary operator $M_{\kappa}\left(\tau_{\alpha}\right)$ and $M_{\kappa}\left(\Phi_{\beta}^{k}\right)$ on $\mathscr{H}_{N}(\kappa)$. We treat $\tau_{\alpha}$ first.

Translations and skew translations were already quantized in [DBDEG] using ideas from geometric quantization. We proceed slightly differently here. A natural way to associate a unitary operator to $\tau_{\alpha}$ would have been to choose the translation operator $U\left(\alpha_{1}, \alpha_{2}\right)=e^{\frac{i}{\hbar}\left(\alpha_{2} Q-\alpha_{1} P\right)}$, restricted to $\mathscr{H}_{N}(\kappa)$. But as we saw in Sect. 2, no $\mathscr{H}_{N}(\kappa)$ is preserved under these operators, unless $\alpha=\left(\frac{m}{N}, \frac{n}{N}\right)$ for some $n, m \in \mathbb{N}$. We therefore need a different approach to quantize irrational translations. Recall first that

$$
U\left(\alpha_{1}, \alpha_{2}\right)=e^{i \frac{\alpha_{1} \alpha_{2}}{2 \hbar}} U\left(\alpha_{1}, 0\right) U\left(0, \alpha_{2}\right)
$$

This suggests quantizing $\tau_{\left(\alpha_{1}, 0\right)}$ and $\tau_{\left(0, \alpha_{2}\right)}$ separately yielding $M_{\kappa}\left(\tau_{\left(\alpha_{1}, 0\right)}\right)$ and $M_{\kappa}\left(\tau_{\left(0, \alpha_{2}\right)}\right)$ and then defining

$$
M_{\kappa}\left(\tau_{\alpha}\right)=e^{l \frac{\alpha_{1} \alpha_{2}}{2 \hbar}} M_{\kappa}\left(\tau_{\left(\alpha_{1}, 0\right)}\right) M_{\kappa}\left(\tau_{\left(0, \alpha_{2}\right)}\right) .
$$

This is how we will proceed. Recall now from (2.11) that

$$
U\left(0, \frac{n}{N}\right) e_{j}^{\kappa}=e^{i\left(\kappa_{2}+2 \pi j\right) \frac{n}{N}} e_{j}^{\kappa} .
$$

In other words, the $e_{j}^{\kappa}, j \in\{0, \ldots, N-1\}$ are a basis of eigenvectors for the translations $U\left(0, \frac{n}{N}\right)$. This suggests the definition

$$
M_{\kappa}\left(\tau_{\left(0, \alpha_{2}\right)}\right) e_{j}^{\kappa}=e^{i\left(\kappa_{2}+2 \pi j\right) \alpha_{2}} e_{j}^{\kappa} .
$$

$M_{\kappa}\left(\tau_{\left(\alpha_{1}, 0\right)}\right)$ is defined similarly by noting that $U\left(\frac{n}{N}, 0\right)$ is diagonal in the momentum representation.

To establish the equipartition result, the last ingredient needed is an Egorov type theorem. 
Proposition 4.1. There exists a $G_{\delta}$ dense subset $\mathscr{D}$ of $T^{(2)}$ with the property that $\forall \alpha \in \mathscr{D}, \exists\left(N_{k}\right)_{k \in \mathbb{N}}$ such that $\forall f \in C^{\infty}\left(T^{(2)}\right), \exists C$ so that

$$
\left\|M_{\kappa}\left(\tau_{\alpha}\right)^{*} O p_{\kappa}^{W}(f) M_{\kappa}\left(\tau_{\alpha}\right)-O p_{\kappa}^{W}\left(f \circ \tau_{\alpha}\right)\right\|_{\mathscr{L}\left(\mathscr{H}_{N}(\kappa)\right)} \leqq \frac{C}{N_{k}} .
$$

The proof is given below. We can now write and prove the equipartition result for the translations.

Theorem 4.2. Write $M_{\kappa}\left(\tau_{\alpha}\right) \phi_{j}^{N}=\lambda_{j}^{N} \phi_{j}^{N}$ for the eigenfunctions and the eigenvalues of $M_{\kappa}\left(\tau_{\alpha}\right)$, where $\alpha$ belongs to the $G_{\delta}$ dense set $\mathscr{D}$ of Proposition 4.1. For all $f \in C^{\infty}\left(T^{(2)}\right)$ and for all maps

$$
j: N_{k} \in \mathbb{N} \longrightarrow j\left(N_{k}\right) \in\left\{1, \ldots, N_{k}\right\},
$$

we have

$$
\lim _{k \rightarrow \infty}\left\langle\phi_{j\left(N_{k}\right)}^{N_{k}}, O p_{\kappa}^{W}(f) \phi_{j\left(N_{k}\right)}^{N_{k}}\right\rangle_{(\kappa, N)}=\int_{T^{(2)}} f \frac{d q d p}{a b} .
$$

Proof. From the unitary of $M_{\kappa}\left(\tau_{\alpha}\right)$, it follows that $\forall T \in \mathbb{N}^{*}$

$$
\begin{aligned}
& \left|\left\langle\phi_{j\left(N_{k}\right)}^{N_{k}}, O p_{\kappa}^{W}(f) \phi_{j\left(N_{k}\right)}^{N_{k}}\right\rangle_{(\kappa, N)}-\int_{T^{(2)}} f \frac{d q d p}{a b}\right| \\
& =\left|\left\langle\phi_{j\left(N_{k}\right)}^{N_{k}}, \frac{1}{T} \sum_{l=1}^{T} M_{\kappa}\left(\tau_{\alpha}\right)^{* l} O p_{\kappa}^{W}(f) M_{\kappa}\left(\tau_{\alpha}\right)^{l} \phi_{j\left(N_{k}\right)}^{N_{k}}\right\rangle_{(\kappa, N)}-\int_{T^{(2)}} f \frac{d q d p}{a b}\right| \\
& \quad \leqq\left|\left\langle\phi_{j\left(N_{k}\right)}^{N_{k}}, O p_{\kappa}^{W}\left[\frac{1}{T} \sum_{l=1}^{T} f \circ \tau_{l \alpha}-\int_{T^{(2)}} f \frac{d q d p}{a b}\right] \phi_{j\left(N_{k}\right)}^{N_{k}}\right\rangle_{(\kappa, N)}\right|+\frac{C_{T}(f)}{N_{k}},
\end{aligned}
$$

where we used Proposition 4.1. Now using Lemma 3.9 and Lemma 3.8 (iv), we have

$$
\begin{aligned}
& \left|\left\langle\phi_{j\left(N_{k}\right)}^{N_{k}}, O p_{\kappa}^{W}(f) \phi_{j\left(N_{k}\right)}^{N_{k}}\right\rangle_{(\kappa, N)}-\int_{T^{(2)}} f \frac{d q d p}{a b}\right| \\
& \quad \leqq\left|\left\langle\phi_{j\left(N_{k}\right)}^{N_{k}}, O p_{\kappa}^{A W}\left[\frac{1}{T} \sum_{l=1}^{T} f \circ \tau_{l \alpha}-\int_{T^{(2)}} f \frac{d q d p}{a b}\right] \phi_{j\left(N_{k}\right)}^{N_{k}}\right\rangle_{(\kappa, N)}\right|+\frac{C_{T}^{\prime}(f)}{N_{k}} \\
& \quad \leqq\left\|\frac{1}{T} \sum_{l=1}^{T} f \circ \tau_{l \alpha}-\int_{T^{(2)}} f \frac{d q d p}{a b}\right\|_{\infty}+\frac{C_{T}^{\prime}(f)}{N_{k}} .
\end{aligned}
$$

Using (4.1), the result follows.

Comparing this to Theorem 1.1, we see this is better in that we can take $E\left(N_{k}\right)=\left\{1, \ldots, N_{k}\right\}$. Of course, the result is subject to the same flaw as the one pointed out in the introduction concerning [DEGI]: the sequence $N_{k}$ depends on $\alpha$. This is all the more unsatisfactory here since the translations $\tau_{\alpha}$ are uniquely ergodic so that there are no other invariant measures for eigenfunctions to concentrate on. The origin of the problem is to be found in the rather weak version of Egorov given 
by Proposition 4.1. It can probably not be improved considerably. If, for example $\alpha=\left(\alpha_{1}, 0\right), \alpha_{1} \notin \mathbb{Q}$, then one can show

$$
\limsup _{N \rightarrow \infty}\left\|M_{\kappa}\left(\tau_{\alpha}\right)^{*} O p_{\kappa}^{W}(f) M_{\kappa}\left(\tau_{\alpha}\right)-O p_{\kappa}^{W}\left(f \circ \tau_{\alpha}\right)\right\|_{\mathscr{L}\left(\mathscr{H}_{N}(\kappa)\right)}=2
$$

if $f(q, p)=e^{2 \pi i(n q-m p)}$.

We now turn to the proof of Proposition 4.1.

Lemma 4.3. Let $\alpha, \alpha^{\prime} \in T^{(2)}$. Then $\exists C>0$ so that $\forall N>0$,

$$
\left\|M_{\kappa}\left(\tau_{\alpha}\right)-M_{\kappa}\left(\tau_{\alpha^{\prime}}\right)\right\|_{\mathscr{L}\left(\mathscr{H}_{N}(\kappa)\right)} \leqq C N\left(\left|\alpha_{1}-\alpha_{1}^{\prime}\right|+\left|\alpha_{2}-\alpha_{2}^{\prime}\right|\right) .
$$

Proof. Note that, for $\psi=\sum_{j=0}^{N-1} c_{j} e_{j}^{\kappa}$ one has, using (4.3),

$$
\left\|\left(M_{\kappa}\left(\tau_{\left(0, \alpha_{2}\right)}\right)-M_{\kappa}\left(\tau_{\left(0, \alpha_{2}^{\prime}\right)}\right)\right) \psi\right\|_{(\kappa, N)} \leqq C N\left|\alpha_{2}-\alpha_{2}^{\prime}\right|\|\psi\|_{(\kappa, N)} .
$$

This, together with a similar argument for $M_{\kappa}\left(\tau_{\left(\alpha_{1}, 0\right)}\right)$ and (4.2) yields the result.

Note that this implies that the map $\alpha \rightarrow M_{\kappa}\left(\tau_{\alpha}\right)$ is continuous.

Proof of Proposition 4.1. The set $\mathscr{D}$ is defined as those $\alpha \in T^{(2)}$ for which $\exists\left(N_{k}\right)_{k \in \mathbb{N}}$, so that $\left|\alpha_{1}-\frac{p_{1, k}}{N_{k}}\right|<\frac{1}{N_{k}^{2}}$ and $\left|\alpha_{2}-\frac{p_{2, k}}{N_{k}}\right|<\frac{1}{N_{k}^{2}}$ have simultaneous solutions for $p_{1, k} \in \mathbb{Z}$ and $p_{2, k} \in \mathbb{Z}$. This is easily seen to be a $G_{\delta}$ set. The proof follows immediately from Lemma 4.3 with $\alpha^{\prime}=\left(\frac{p_{1, k}}{N_{k}}, \frac{p_{2, k}}{N_{k}}\right)$ and the observation that $\forall n, m \in \mathbb{Z}$,

$$
U\left(\frac{n}{N}, \frac{m}{N}\right)^{*} O p^{W}(f) U\left(\frac{n}{N}, \frac{m}{N}\right)=O p^{W}\left(f \circ \tau_{\left(\frac{n}{N}, \frac{m}{N}\right)}\right) .
$$

We end this section with a brief discussion of the skew translations. We will explain in Sect. 6 how to quantize $K=\left(\begin{array}{ll}1 & k \\ 0 & 1\end{array}\right) \in \operatorname{SL}(2, \mathbb{Z})$. Consequently, we define

$$
M_{\kappa}\left(\Phi_{\beta}^{k}\right) \equiv M_{\kappa}\left(\tau_{(0, \beta)}\right) \circ M_{\kappa}(K)
$$

Since the Egorov theorem is exact for $M_{K}(K)$ (see Lemma 6.2), it suffices to use Lemma 4.3 as in the proof of Proposition 4.1 to get an Egorov theorem for all $(\beta, k) \in \mathbb{R} \times \mathbb{Z}$. This yields the desired equipartition result that we won't state explicitly.

\section{Equipartition of the Eigenfunctions}

In this section we prove Theorem 1.1. The hypotheses of the theorem will be assumed throughout. Using the results of Sect. 3, the proof is easily adapted from the proof of Theorem 2.2 and 3.1 in [HMR] and we will not therefore give all details. We start with some preliminary work. The first lemma is a trivality that does not involve any knowledge on the dynamics $\Phi$ or on $M_{\kappa}(\Phi)$. It is in fact a direct consequence of Proposition 3.10. Its analogue in [Z1, CdV, HMR] is not trivial at all and does involve the dynamics. 
Lemma 5.1. $\forall f \in C^{\infty}\left(T^{(2)}\right)$,

$$
\bar{\mu}_{N}(f) \equiv \frac{1}{N} \sum_{j=1}^{N} \mu_{j}^{N}(f) \stackrel{N \rightarrow \infty}{\longrightarrow} \mu(f),
$$

where $\mu_{j}^{N}(f) \equiv\left\langle O p_{\kappa}^{A W}(f) \phi_{j}^{N}, \phi_{j}^{N}\right\rangle$.

Remarks.

(i) It is understood that we choose a $\kappa$ for each $N$. We shall not indicate the $\kappa$ dependence of the $\mu_{j}^{N}$.

(ii) We can write

$$
\mu_{j}^{N}(f)=\int_{T^{(2)}} f(q, p) d \mu_{j}^{N}(q, p)
$$

where $d \mu_{j}^{N}(q, p)=\left|\left\langle q, p, z, \kappa \mid \phi_{j}^{N}\right\rangle\right|^{2} \frac{d q d p}{2 \pi \hbar}$. So the $\mu_{j}^{N}$ are in view of Lemma 3.8 positive probability measures on $T^{(2)}$, absolutely continuous with respect to the Liouville measure.

Proof.

$$
\begin{aligned}
\left|\bar{\mu}_{N}(f)-\mu(f)\right| & =\left|\frac{1}{N} \operatorname{Tr} O p_{\kappa}^{A W}(f)-\mu(f)\right| \\
& \leqq \frac{1}{N}\left|\operatorname{Tr}\left(O p_{\kappa}^{A W}(f)-O p_{\kappa}^{W}(f)\right)\right|+\left|\frac{1}{N} \operatorname{Tr} O p_{\kappa}^{W}(f)-\mu(f)\right| \\
& \leqq\left\|O p_{\kappa}^{A W}(f)-O p_{\kappa}^{W}(f)\right\|_{\mathscr{L}_{\left(\mathscr{H}_{N}(\kappa)\right)}+\left|\frac{1}{N} \operatorname{Tr} O p_{\kappa}^{W}(f)-\mu(f)\right|}
\end{aligned}
$$

The result follows from Lemma 3.9 and Proposition 3.10.

The lemma states that on average the $\left\langle O p_{\kappa}^{A W}(f) \phi_{j}^{N}, \phi_{j}^{N}\right\rangle$ converge to the desired quantity, a fact we already knew for the $\left\langle O p_{\kappa}^{W}(f) \phi_{j}^{N}, \phi_{j}^{N}\right\rangle$ from Proposition 3.10. The core of Theorem 1.1 is hidden in the following proposition. To establish it, the dynamics $\Phi$ intervenes through its ergodicity and the Egorov theorem (i.e. Eq. (1.1)).

Proposition 5.2. $\forall f \in C^{\infty}\left(T^{(2)}\right)$ and $\forall \varepsilon>0$,

$$
\lim _{N \rightarrow \infty} \frac{\#\left\{j ;\left|\mu_{j}^{N}(f)-\mu(f)\right|<\varepsilon\right\}}{N}=1 .
$$

Proof. For $f \in C^{\infty}\left(T^{(2)}\right)$ and for $p \in \mathbb{N}^{*}$ introduce the time average

$$
f_{p}=\frac{1}{p} \sum_{k=1}^{p} f \circ A^{k} \text {. }
$$

The proof is a direct adaptation from the one in [HMR]. Indeed it is based, as in Sect. 4, on four observations. First,

$$
\mu_{j}^{N}(f)-\mu(f)=\left\langle\phi_{j}^{N}, O p_{\kappa}^{A W}(f-\mu(f)) \phi_{j}^{N}\right\rangle
$$


is close to $\left\langle\phi_{j}^{N}, O p_{\kappa}^{W}(f-\mu(f)) \phi_{j}^{N}\right\rangle$ for large $N$ thanks to Lemma 3.9. Next, since the $\phi_{j}^{N}$ are eigenfunctions of $M_{\kappa}(\Phi)$ (stationary states), we have

$$
\left\langle\phi_{j}^{N}, O p_{\kappa}^{W}(f-\mu(f)) \phi_{j}^{N}\right\rangle=\frac{1}{p} \sum_{k=1}^{p}\left\langle\phi_{j}^{N}, M_{\kappa}(\Phi)^{* k} O p_{\kappa}^{W}(f-\mu(f)) M_{\kappa}(\Phi)^{k} \phi_{j}^{N}\right\rangle .
$$

The Egorov hypothesis 1.1 then implies that, for large $N$, the right-hand side is close to $\left\langle\phi_{j}^{N}, O p_{\kappa}^{W}\left(f_{p}-\mu(f)\right) \phi_{j}^{N}\right\rangle$. Finally, we will use ergodicity, i.e. $f_{p} \rightarrow \mu(f)$ together with Lemma 5.1, to conclude that, for large $p$ and $N,\left\langle\phi_{j}^{N}, O p_{\kappa}^{W}\left(f_{p}-\right.\right.$ $\left.\mu(f)) \phi_{j}^{N}\right\rangle$ is small, at least for "many" $j$. The detailed argument is analogous to the one in [HMR] and we skip it.

A direct consequence of Proposition 5.2 is the following corollary which is essentially the statement of Theorem 1.1, except for the dependence of $E(N)$ on $f$.

Corollary 5.3. For all $f \in C^{\infty}\left(T^{(2)}\right)$ for all $N$, there exists a subset $E(N, f)$ of $\{1, \ldots, N\}$ satisfying

(i)

$$
\lim _{N \rightarrow \infty} \frac{\# E(N, f)}{N}=1
$$

(ii) for all maps $j: N \in \mathbb{N} \longrightarrow j(N) \in E(N, f)$

$$
\lim _{N \rightarrow \infty}\left\langle O p_{\kappa}^{W}(f) \phi_{j(N)}^{N}, \phi_{j(N)}^{N}\right\rangle_{(\kappa, N)}=\mu(f),
$$

and

$$
\lim _{N \rightarrow \infty}\left\langle O p_{\kappa}^{A W}(f) \phi_{j(N)}^{N}, \phi_{j(N)}^{N}\right\rangle_{(\kappa, N)}=\mu(f),
$$

uniformly with respect to the sequence $j$.

The proofs of Corollary 5.3 and Theorem 1.1 use completely analogous arguments to the ones in [HMR] and we omit them.

\section{Quantization of the Automorphisms of $T^{(2)}$}

The results of Sect. 3 allow us to quantize any globally Hamiltonian flow on $T^{(2)}$ as follows. If $H \in C^{\infty}\left(T^{(2)}\right)$ is the classical Hamiltonian of the flow then the corresponding unitary one-parameter group on $\mathscr{H}_{N}(\kappa)$ is $\exp \left(-i \frac{t}{\hbar} O p_{\kappa}^{W}(H)\right)$. But for discrete maps such as the automorphisms of the torus, this approach does not work. The framework we set up nevertheless provides an easy quantization for them, as we now show. Any quadratic Hamiltonian $H(q, p)=\alpha q^{2}+\beta q p+\gamma p^{2}$ on $\mathbb{R}^{2}$ generates a linear flow $\Phi_{t}$ : for all $t, \Phi_{t} \in S L(2, \mathbb{R})$. The corresponding Weyl quantized operators generate a unitary one-parameter group on $L^{2}(\mathbb{R})$. Explicitly, one obtains a map $A \in S L(2, \mathbb{R}) \longrightarrow M(A) \in \mathfrak{U}\left(L^{2}(\mathbb{R})\right)$, where for $a_{2} \neq 0$,

with

$$
[M(A) \psi](x)=\left(\frac{i}{2 \pi \hbar a_{2}}\right)^{1 / 2} \int e^{\frac{l}{\hbar} S(x, y)} \psi(y) d y
$$

$$
S(x, y)=\frac{1}{2} \frac{a_{4}}{a_{2}} x^{2}-\frac{1}{a^{2}} x y+\frac{1}{2} \frac{a_{1}}{a_{2}} y^{2},
$$


where $A=\left(\begin{array}{ll}a_{1} & a_{2} \\ a_{3} & a_{4}\end{array}\right)$. The $M(A)$ are uniquely determined up to a phase, which can be fixed by group-theoretic consideration that won't concern us here $[\mathrm{F}]$. A crucial property of the $M(A)$ is:

$$
M(A) U(q, p) M(A)^{*}=U\left(A\left(\begin{array}{c}
q \\
p
\end{array}\right)\right) .
$$

We shall refer to $M(A)$ as the quantum propagator associated to $A$.

The subgroup of $\operatorname{SL}(2, \mathbb{R})$ which leaves $\Gamma$ (see Sect. 2) invariant is made up of elements of the form

$$
A=\left(\begin{array}{cc}
\alpha & \beta \frac{a}{b} \\
\gamma \frac{b}{a} & \delta
\end{array}\right)
$$

with $\alpha \delta-\beta \gamma=1$ and $(\alpha, \delta, \beta, \gamma) \in \mathbb{Z}^{4}$. It is diffeomorphic to $\operatorname{SL}(2, \mathbb{Z})$, and we shall refer to the $A$ in $(6.3)$ as belonging to $S L(2, \mathbb{Z})$ with some abuse of language. Any $A$ of the form (6.3) acts naturally on $T^{(2)}=\mathbb{R}^{2} / \Gamma$ as an area-preserving map. It is well known [AA, CFS] that these transformations are globally hyperbolic dynamical systems and hence ergodic iff $|\operatorname{Tr} A|>2$.

We now show how to associate to any $A \in S L(2, \mathbb{Z})$ a unitary operator on (some) $\mathscr{H}_{N}(\kappa)$. Clearly these $A$ do not belong to one-parameter groups generated by a Hamiltonian. Hence we cannot use Weyl-quantization as above. Instead, we simply study the restriction of $M(A)$ in $(6.1)$ to $\mathscr{H}_{N}(\kappa)$. We have

Lemma 6.1.

$$
M(A) \mathscr{H}_{N}(\kappa) \subset \mathscr{H}_{N}\left(\kappa^{\prime}\right)
$$

where

$$
\left(\begin{array}{l}
\kappa_{2}^{\prime} \\
\kappa_{1}^{\prime}
\end{array}\right)=A\left(\begin{array}{l}
\kappa_{2} \\
\kappa_{1}
\end{array}\right)+\pi N\left(\begin{array}{l}
\alpha \beta / b \\
\gamma \delta / a
\end{array}\right)+\left(\begin{array}{l}
\bmod \left(\frac{2 \pi}{b}\right) \\
\bmod \left(\frac{2 \pi}{a}\right)
\end{array}\right) .
$$

Proof. Equation (6.2) implies

$$
U(n a, m b) M(A)=M(A) U\left(n^{\prime} a, m^{\prime} b\right),
$$

where $\left(\begin{array}{c}n^{\prime} a \\ m^{\prime} b\end{array}\right)=A^{-1}\left(\begin{array}{c}n a \\ m b\end{array}\right)$, so $\left(\begin{array}{c}n^{\prime} \\ m^{\prime}\end{array}\right)=\left(\begin{array}{cc}\delta & -\beta \\ -\gamma & \alpha\end{array}\right)\left(\begin{array}{c}n \\ m\end{array}\right)$. Then, using (2.1)-(2.3), for all $\psi \in \mathscr{H}_{N}(\kappa)$, we have that

$$
U(n a, m b) M(A) \psi=e^{i \pi N n^{\prime} m^{\prime}} e^{-i\left(\kappa_{1} n^{\prime} a-\kappa_{2} m^{\prime} b\right)} M(A) \psi
$$

Introducing the canonical symplectic form on $T^{(2)}: \omega:=d q \wedge d p$, we remark that $\kappa_{1} n^{\prime} a-\kappa_{2} m^{\prime} b=\omega\left(\left(n^{\prime} a, m^{\prime} b\right),\left(\kappa_{2}, \kappa_{1}\right)\right)$. As a result, since $\operatorname{det} A^{-1}=1$,

$$
\kappa_{1} n^{\prime} a-\kappa_{2} m^{\prime} b=\kappa_{1}^{\prime \prime} n a-\kappa_{2}^{\prime \prime} m b,
$$

where $\left(\begin{array}{c}\kappa_{2}^{\prime \prime} \\ \kappa_{1}^{\prime \prime}\end{array}\right)=A\left(\begin{array}{l}\kappa_{2} \\ \kappa_{1}\end{array}\right)$ and

$$
U(n a, m b) M(A) \psi=e^{i \pi N\left[-\gamma \delta n^{2}-\alpha \beta m^{2}+(\alpha \delta+\beta \gamma) n m\right]} e^{-i\left(\kappa_{1}^{\prime \prime} n a-\kappa_{2}^{\prime \prime} m b\right)} M(A) \psi
$$


Since $-\gamma \delta n^{2}-\alpha \beta m^{2}+(\alpha \delta+\beta \gamma) n m=\gamma \delta n+\alpha \beta m+m n(\bmod 2)$, we have

$$
U(n a, m b) M(A) \psi=e^{i \pi N n m} e^{-i\left[\left(\kappa_{1}^{\prime \prime}+\pi N \frac{\gamma \delta}{a}\right) n a-\left(\kappa_{2}^{\prime \prime}+\pi N \frac{\alpha \beta}{b}\right) m b\right]} M(A) \psi .
$$

Hence, since $\kappa_{1}, \kappa_{2}$ are defined $\bmod \left(\frac{2 \pi}{a}\right), \bmod \left(\frac{2 \pi}{b}\right)$ (respectively) and the relation modulo is preserved by $A,(6.4)$ follows.

Given $A$ with $|\operatorname{Tr} A|>2$ there exists for each $N$ a $\kappa \in\left[0, \frac{2 \pi}{a}\left[\times\left[0, \frac{2 \pi}{b}\right.\right.\right.$ [ so that $\kappa^{\prime}=\kappa$. This choice can be made independent of $N$ iff $A$ is of the form

$$
A=\left(\begin{array}{cc}
\operatorname{even} & \operatorname{odd} \frac{a}{b} \\
\operatorname{odd} \frac{b}{a} & \text { even }
\end{array}\right) \quad \text { or }\left(\begin{array}{cc}
\operatorname{odd} & \operatorname{even} \frac{a}{b} \\
\operatorname{even} \frac{b}{a} & \text { odd }
\end{array}\right),
$$

in which case one can take $\kappa=0$. This is the case studied in [HB]. Otherwise, there exists at least one choice for all even $N$ and at least one choice for odd $N$, different one from the other. The choices are unique if $\operatorname{Tr} A=3$. When $A, N, \kappa$ are fixed so that $M(A) \mathscr{H}_{N}(\kappa) \subset \mathscr{H}_{N}(\kappa)$, we shall write $M_{\kappa}(A)$ for the restriction of $M(A)$ to $\mathscr{H}_{N}(\kappa)$; we shall refer to $M_{\kappa}(A)$ as the quantum propagator associated to the area-preserving map $A \in S L(2, \mathbb{Z})$ on $T^{(2)}$.

It remains to show $M_{\kappa}(A)$ is unitary. But this is obvious since it is the restriction of $M(A)$ to one fiber of the direct integral (2.8).

We can now introduce the last ingredient that is needed in order to apply Theorem 1.1 to $\Phi=A$.

Lemma 6.2. Let $f \in C^{\infty}\left(T^{(2)}\right)$, then

$$
O p_{\kappa}^{W}(f \circ A)=M_{\kappa}(A)^{*} O p_{\kappa}^{W}(f) M_{\kappa}(A) .
$$

Proof. Since $O p^{W}(f \circ A)=M(A)^{*} O p^{W}(f) M(A)[\mathrm{F}],(6.6)$ is obvious, because $M_{\kappa}(A)$ and $O p_{\kappa}^{W}(f)$ are simply obtained by restricting $M(A)$ and $O p^{W}(f)$ to $\mathscr{H}_{N}(\kappa)$.

Note that our proof of the equipartition result for $M_{\kappa}(A)$ does not use the explicit form of the $M_{\kappa}(A)$. Viewed as matrices on $\mathbb{C}^{N}$, the latter are indeed rather complicated [HB, DE, DEGI] unless

$$
A=\left(\begin{array}{cc}
2 g & 1 \\
4 g^{2}-1 & 2 g
\end{array}\right), \quad g \in \mathbb{N},
$$

which are the matrices studied in $[\mathrm{HB}]$ and for which the equipartition result is proven in [DEGI].

As a final remark, we point out that the Wigner function $W_{\kappa}\left(\phi_{j}^{N}, \phi_{j}^{N}\right)$ of any eigenfunction of $M_{\kappa}(A)$ is invariant, i.e.

$$
W_{\kappa}\left(\phi_{j}^{N}, \phi_{j}^{N}\right) \circ A^{-1}=W_{\kappa}\left(\phi_{j}^{N}, \phi_{j}^{N}\right) .
$$

This is a direct consequence of (3.7) and (6.6). This property is not shared by the polynomial Weyl symbol, since $\mathscr{I}_{N}$ is not invariant under $A$.

Acknowledgements. The authors would like to thank Mirko Degli Esposti and Sandro Graffi for many helpful conversations and for explaining their work to them. Stephan De Bièvre would like to thank the Department of Mathematics of the University of Bologna, where part of this work was performed, for its hospitality. 


\section{References}

[AA] Arnold, V.I., Avez, A.: Ergodic problems in classical mechanics. New York: W.A. Benjamin, 1968

[AB] Agam, O., Brenner, N.: Semi-classical Wigner functions for quantum maps on the torus. Preprint Technion-Phys-94

[BV] Balazs, N.L., Voros, A.: The quantized Baker's transformation. Ann. Phys. 190, 1-31 (1989)

[CdV] Colin de Verdière, Y.: Ergodicité et fonctions propres du Laplacien. Commun. Math. Phys. 102, 497-502 (1985)

[CFS] Cornfeld, I.P., Fomin, S.V., Sinai, Ya.G.: Ergodic theory. Berlin: Springer Verlag, 1982

[CR] Combescure, M., Robert, D.: Distribution of matrix elements and level spacings for classically chaotic systems. Ann. Inst. H. Poincaré 61, 4, 443-483 (1994)

[DB] De Bièvre, S.: Oscillator eigenstates concentrated on classical trajectories. J. Phys. A. 25, 3399-3418 (1992)

[DBDEG] De Bièvre, S., Degli Esposti, M., Giachetti, R.: Quantization of a class of piecewise affine transformations on the torus. Preprint, 1994, Commun. Math. Phys., to appear

[DBG] De Bièvre, S., Gonzalez, J.A.: Coherent states on tori. In: "Quantization and coherent states methods." Proceedings of the 11th Work-shop on Geometrical Methods in Mathematical Physics-Bialystoc 1992, Ali, S.T., Ladanov, I.M., Odzijewicz, A. (eds.) Singapore: World Scientific, 1993

[DE] Degli Esposti, M.: Quantization of the orientation preserving automorphisms of the torus. Ann. Inst. H. Poincaré 58, 323-341 (1993)

[DEGI] Degli Esposti, M., Graffi, S., Isola, S.: Stochastic properties of the quantum Arnold cat in the classical limit. Commun. Math. Phys. 167, 471-509 (1995)

[FGBV] Figueroa, H., Garcia-Bondia, J.M., Varilly, J.C.: Moyal quantization with compact symmetry groups and noncommutative harmonic analysis. J. Math. Phys. 31, 2664-2671 (1990)

[F] Folland, G.: Harmonic analysis in phase space. Princeton, NJ: Princeton University Press, 1988

[GL] Gérard, P., Leichtnam, E.: Ergodic properties of the eigenfunctions for the Dirichlet problem. Duke. Math. J. 71, 2, 559-607 (1993)

[HB] Hannay, J.H., Berry, M.V.: Quantization of linear maps-Fresnel diffraction by a periodic grating. Physica D. 1, 267-291 (1980)

[HMR] Helffer, B., Martinez, A., Robert, D.: Ergodicité et limite semi-classique. Commun. Math. Phys. 109, 313-326 (1987)

[HOSW] Hillery, M., O'Connel, R.F., Scully, M.O., Wigner, E.P.: Distribution functions in Physics: Fundamentals. Phys. Rep. 106, 121-167 (1984)

[LV] Leboeuf, P., Voros, A.: Chaos-revealing multiplicative representation of quantum eigenstates. J. Phys. A23, 1765-1774 (1990)

[M] Mané, R.: Ergodic theory and differentiable dynamics. Berlin: Springer Verlag, 1987

[Pe] Perelomov, A.: Generalized coherent states and their applications. Berlin, Heidelberg, New York: Springer, 1985

[Ro] Robert, D.: Autour de l'approximation semi-classique. Birkhäuser, 1987

[RS] Reed, M., Simon, B.: Analysis of operators IV. New York: Academic Press, 1978

[S] Saraceno, M.: Classical structures in the quantized Baker transformation. Ann. Phys. 199, 37-60 (1990)

[Sa] Sarnak, P.: Arithmetic Quantum Chaos. Tel Aviv Lectures, 1993

[Sc] Schnirelman, A.: Ergodic properties of eigenfunctions. Usp. Math. Nauk 29, 181-182 (1974)

[Si] Sinai, Ya.G.: Topics in ergodic theory. Princeton, NJ: Princeton University Press, 1994

[Z1] Zelditch, S.: Uniform distribution of the eigenfunctions on compact hyperbolic surfaces. Duke. Math. J. 55, 919-941 (1987)

[Z2] Zelditch, S.: Quantum ergodicity of quantized contact transformations and ergodic symplectic toral automorphisms. Preprint 1994 
